Journal of Applied Fluid Mechanics, Vol. 6, No. 3, pp. 311-320, 2013.

Available online at www.jafmonline.net, ISSN 1735-3572, EISSN 1735-3645.

DOI: $10.36884 /$ jafm.6.03.19578

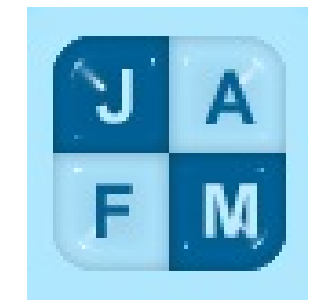

\title{
Applied Filtered Density Function
}

\author{
S. L. Yilmaz ${ }^{1}$, N. Ansari ${ }^{1,2}$, P. H. Pisciuneri ${ }^{1}$, M. B. Nik ${ }^{1}$, C. C. Otis ${ }^{1}$, and P. Givi ${ }^{1 \dagger}$ \\ ${ }^{1}$ University of Pittsburgh, Pittsburgh, PA, 15260, USA \\ 2 ANSYS Inc., Canonsburg, PA, 15317, USA \\ $\dagger$ Corresponding Author Email: pgivi@ pitt.edu
}

(Recieved December 16, 2012; accepted March 7, 2013)

\begin{abstract}
An overview is presented of recent advances in the filtered density function (FDF) modeling and simulation of turbulent combustion. The review is focused on the developments that have facilitated the FDF to be broadly applied in large eddy simulation (LES) of practical flows. These are primarily the development of a new Lagrangian Monte Carlo solver for the FDF, and the implementation of this solver on Eulerian domains portrayed by unstructured grids. With these developments, it is now much easier to apply FDF for predictions of reacting flows in complex geometrical configurations.
\end{abstract}

Keywords: Turbulence, Combustion, Large eddy simulation, Filtered density function

\section{INTRODUCTION}

The filtered density function (FDF); including its mass weighted form, the filtered mass density function (FMDF), is now considered as one of the most effective means of conducting large eddy simulation (LES) in turbulent combustion Givi (2006). The FDF is essentially the counterpart of the probability density function (PDF) methods in Reynolds-averaged Navier-Stokes (RANS) simulations Pope (2013). In its stand-alone form, the FDF must account for the joint statistics of all of the relevant physical variables. The most sophisticated FDF closure available to-date is the frequency-velocityscalar FMDF (FVS-FMDF) Sheikhi et al. (2009). A simpler version (VS-FMDF) that does not include the subgrid-scale (SGS) frequency also exists (Sheikhi et al. 2003, 2007). Hydrodynamic closure in incompressible, non-reacting flows has been successfully achieved via the velocity-FDF (V-FDF) Gicquel et al. (2002). The scalar FDF (S-FDF and S-FMDF) is the most widely used form and considers only the scalar field. This is the most elementary form of the FDF that we first introduced (Colucci et al. 1998; Garrick et al. 1999; Jaberi et al. 1999). The first LES of a hydrocarbon flame, namely the Sandia-Darmstadt piloted diffusion flame (Barlow and Frank 1998; Sandia National Laboratories 2013), has been conducted via both S-FMDF (Sheikhi et al. 2005) and VS-FMDF (Nik, Yilmaz, Givi et al. 2010; Nik, Yilmaz, Sheikhi et al. 2010). The FDF has also been successful in predicting the more complex field of the bluff- body Sandia-Sydney flame Dally et al. $(1998,2003)$ and a premixed Bunsen burner Chen et al. (1996) as reported in Drozda et al. (2007) and Yilmaz et al. (2010), respectively.

The original work of Colucci et al. (1998) provides the first demonstration of a transported FDF. Since then, this methodology has experienced widespread usage, and is now regarded as one of the most effective and popular means of LES worldwide. Some of the most important contributions to the FDF by others are in its basic implementation (Afshari et al. 2008; Carrara and DesJardin 2006; Chen 2007; Drozda et al. 2008; Heinz 2003b; James et al. 2007; Jones and Navarro-Martinez 2007; Jones et al. 2007; McDermott and Pope 2007; Mustata et al. 2006; Raman and Pitsch 2005, 2007; Raman et al. 2005; van Vliet et al. 2005; Zhou and Pereira 2000), fine-tuning of its sub-closures (Cha and Trouillet 2003; Heinz 2007; Réveillon and Vervisch 1998) and its validation via laboratory experiments (Rajagopalan and Tong 2003; Tong 2001; van Vliet et al. 2005; Wang and Tong 2002, 2005; Wang et al. 2004). For a review of the state of progress in FDF modeling we refer to Ansari et al. (2011). For a comprehensive understanding of the FDF, we refer serious readers to several dissertations (Ansari 2012; Colucci 1998; Drozda 2005; Gicquel 2001; Nik 2012; Pisciuneri 2008; Sheikhi 2005; Yilmaz 2008).

In this article, we provide a review of recent developments which facilitate FDF to be applied to LES of complex turbulent reacting flows. The phrase "complex" 
refers to both the chemical kinetics and the geometrical flow configuration. These developments are: (1) construction of an "irregularly portioned" Lagrangian Monte Carlo FDF simulator, and (2) implementation of the Monte Carlo simulator on Eulerian domains portrayed by unstructured grids. The former allows efficient FDF simulations on massively parallel platforms, and the latter facilitates simulation of flows in complex configurations. With these developments, it is now much easier to conduct LES of complex turbulent reacting flows.

\section{FDF FORMUlation AND MODEL- ING}

The primary transport variables in reactive flows are the fluid density $\rho(\mathbf{x}, t)$, the velocity vector $u_{i}(\mathbf{x}, t), i=1,2,3$ along the $x_{i}$ direction, the specific enthalpy $h(\mathbf{x}, t)$, the pressure $p(\mathbf{x}, t)$, and the mass fractions of $N_{s}$ species, $Y_{\alpha}(\mathbf{x}, t)\left(\alpha=1,2, \ldots, N_{s}\right)$, where $\mathbf{x} \equiv x_{i}(i=1,2,3)$ and $t$ denote space and time, respectively. Implementation of LES involves the use of the spatial filtering operation (Geurts 2004; Sagaut 2010)

$$
\langle Q(\mathbf{x}, t)\rangle=\int_{-\infty}^{+\infty} Q\left(\mathbf{x}^{\prime}, t\right) \mathcal{H}\left(\mathbf{x}^{\prime}, \mathbf{x}\right) d \mathbf{x}^{\prime}
$$

where $\mathcal{H}$ denotes the filter function of width $\Delta_{\mathcal{H}}$, and $\langle Q(\mathbf{x}, t)\rangle$ represents the filtered value of the transport variable $Q(\mathbf{x}, t)$. In reacting flows, it is convenient to consider the Favre filtered quantity, $\langle Q(\mathbf{x}, t)\rangle_{L}=\langle\rho Q\rangle /\langle\rho\rangle$. We consider spatially invariant and localized filter functions, $\mathcal{H}\left(\mathbf{x}^{\prime}, \mathbf{x}\right) \equiv \mathcal{H}\left(\mathbf{x}^{\prime}-\mathbf{x}\right)$ with the properties (Ghosal and Moin 1995) $\mathcal{H}(\mathbf{x})=\mathcal{H}(-\mathbf{x})$, and $\int_{-\infty}^{\infty} \mathcal{H}(\mathbf{x}) d \mathbf{x}=1$. We consider positive filter functions Vreman et al. (1994) for which all the moments $\int_{-\infty}^{\infty} x^{m} \mathcal{H}(x) d x$ exist for $m \geq 0$. The transport variables satisfy the conservation equations of mass, momentum, energy and species mass fractions Williams (1985). The filtered form of these equations are:

$$
\frac{\partial\langle\rho\rangle}{\partial t}+\frac{\partial\langle\rho\rangle\left\langle u_{i}\right\rangle_{L}}{\partial x_{i}}=0
$$

$$
\begin{aligned}
\frac{\partial\langle\rho\rangle\left\langle u_{j}\right\rangle_{L}}{\partial t} & +\frac{\partial\langle\rho\rangle\left\langle u_{i}\right\rangle_{L}\left\langle u_{j}\right\rangle_{L}}{\partial x_{i}}= \\
& -\frac{\partial\langle p\rangle}{\partial x_{j}}+\frac{\partial\left\langle\tau_{i j}\right\rangle}{\partial x_{i}}-\frac{\partial T_{i j}}{\partial x_{i}},
\end{aligned}
$$

$$
\begin{aligned}
\frac{\partial\langle\rho\rangle\left\langle\phi_{\alpha}\right\rangle_{L}}{\partial t} & +\frac{\partial\langle\rho\rangle\left\langle u_{i}\right\rangle_{L}\left\langle\phi_{\alpha}\right\rangle_{L}}{\partial x_{i}}= \\
& -\frac{\partial\left\langle J_{i}^{\alpha}\right\rangle}{\partial x_{i}}-\frac{\partial M_{i}^{\alpha}}{\partial x_{i}}+\left\langle\rho S_{\alpha}\right\rangle,
\end{aligned}
$$

where $\tau_{i j}$ and $J_{i}^{\alpha}$ denote the viscous stress tensor and the scalar fluxes, respectively. In Eq. (4), $S_{\alpha}$ denotes the source term, and this equation represents transport of the species mass fractions and enthalpy in a common form with $\phi_{\alpha} \equiv Y_{\alpha}, \alpha=1,2, \ldots, N_{s}, \phi_{\sigma} \equiv$ $h, \sigma=N_{s}+1$. The SGS closure problem is associated with $T_{i j}=\langle\rho\rangle\left(\left\langle u_{i} u_{j}\right\rangle_{L}-\left\langle u_{i}\right\rangle_{L}\left\langle u_{j}\right\rangle_{L}\right), M_{i}^{\alpha}=$ $\langle\rho\rangle\left(\left\langle u_{i} \phi_{\alpha}\right\rangle_{L}-\left\langle u_{i}\right\rangle_{L}\left\langle\phi_{\alpha}\right\rangle_{L}\right)$, and $\left\langle\rho S_{\alpha}\right\rangle$. The FDF provides an effective means for this closure. For the scalars' array $\phi(\mathbf{x}, t)$ and the velocity field, $u(\mathbf{x}, t)$, the SGS statistical information is included in the joint velocity-scalar filtered mass density function (VS-FMDF), denoted by $\mathcal{F}(v, \psi, \mathbf{x}, t)$, where $(v, \psi)$ denote the probability-space for the $(u, \phi)$ fields. The exact transport equation for this FDF is (Sheikhi et al. 2007):

$$
\begin{aligned}
\frac{\partial \mathcal{F}}{\partial t}+\frac{\partial\left(v_{k} \mathcal{F}\right)}{\partial x_{k}} & =\frac{\partial}{\partial v_{k}}\left[\left\langle\frac{1}{\rho} \frac{\partial p}{\partial x_{k}} \mid v, \psi\right\rangle \mathcal{F}\right] \\
& +\frac{\partial}{\partial \psi_{\alpha}}\left[\left\langle\frac{1}{\rho} \frac{\partial J_{j}^{\alpha}}{\partial x_{j}} \mid v, \psi\right\rangle \mathcal{F}\right] \\
& -\frac{\partial}{\partial v_{k}}\left[\left\langle\frac{1}{\rho} \frac{\partial \tau_{k j}}{\partial x_{j}} \mid v, \psi\right\rangle \mathcal{F}\right] \\
& -\frac{\partial}{\partial \psi_{\alpha}}\left[S_{\alpha}(\psi) \mathcal{F}\right],
\end{aligned}
$$

where $\langle\mid\rangle$ denotes the conditional filtered values. As Eq. (5) shows, the effects of SGS convection and combustion are in closed forms. However, all of the terms involving conditional filtered values require closure. The marginal FMDF of the scalar (S-FMDF) field, $\mathcal{F}_{\phi}(\psi, \mathbf{x}, t)$, is obtained by integration of the VS-FMDF over the velocity domain:

$$
\begin{aligned}
\frac{\partial \mathcal{F}_{\phi}}{\partial t} & +\frac{\partial\left[\left\langle u_{i}(\mathbf{x}, t) \mid \psi\right\rangle \mathcal{F}_{\phi}\right]}{\partial x_{i}} \\
& =\frac{\partial}{\partial \psi_{\alpha}}\left[\left\langle\frac{1}{\rho} \frac{\partial J_{j}^{\alpha}}{\partial x_{j}} \mid \psi\right\rangle \mathcal{F}_{\phi}\right] \\
& -\frac{\partial}{\partial \psi_{\alpha}}\left[S_{\alpha}(\psi) \mathcal{F}_{\phi}\right]
\end{aligned}
$$

Again, the effects of chemical reaction appear in a closed form. However, in this case, the SGS convection (second term on the left-hand side) requires closure. This approach has been the most popular among other investigators using FDF (Afshari et al. 2008; Carrara and DesJardin 2006; Cha and Trouillet 2003; Chen 2007; Drozda et al. 2008; Heinz 2003b; James et al. 2007; Jones and Navarro-Martinez 2007; Jones et al. 2007; McDermott and Pope 2007; Mustata et al. 2006; Raman and Pitsch 2005, 2007; Raman et al. 2005; Réveillon and Vervisch 1998; van Vliet et al. 2005; Zhou and Pereira 2000).

The SGS closures have been primarily based on modeled stochastic differential equations (SDEs) for each of the transport variables. These SDEs must account for all of the physics of turbulent combustion; including scalar mixing, chemistry, exothermicity, dilatation, and dissipation. It is straightforward to realize the capability of the FDF in that it accounts for all of the processes involving direct correlations of the transported variables. For example, there is no need for additional closures for turbulent-chemistry interactions and/or velocity-scalar correlations. A system of modeled SDEs (Gikhman and Skorokhod 1972; Karlin and Taylor 1981) based on the stochastic diffusion process (Stratonovich 1963) has proven effective for the FDF closure. The coefficients in the modeled Langevin equation will be set in such a way that the resulting Fokker-Planck equation Risken (1989) defines the modeled FDF transport equation, and thus the SGS closures. In particular, the generalized Langevin model (GLM) (Dreeben and Pope 1997; Haworth and 
Pope 1986; Pope 1994a,b) combined with the linear mean square estimation (LMSE) (Borghi 1988; Dopazo 1994) has proven to be effective. This is described by Sheikhi et al. (2007):

$$
\begin{aligned}
\mathrm{d} x_{i}^{+} & =u_{i}^{+} \mathrm{d} t+\sqrt{\frac{2 \mu}{\langle\rho\rangle}} \mathrm{d} W_{i}, \\
\mathrm{~d} u_{i}^{+} & =-\frac{1}{\langle\rho\rangle} \frac{\partial\langle p\rangle}{\partial x_{i}} \mathrm{~d} t \\
& +\frac{2}{\langle\rho\rangle} \frac{\partial}{\partial x_{j}}\left(\mu \frac{\partial\left\langle u_{i}\right\rangle_{L}}{\partial x_{j}}\right) \mathrm{d} t \\
& +\frac{1}{\langle\rho\rangle} \frac{\partial}{\partial x_{j}}\left(\mu \frac{\partial\left\langle u_{j}\right\rangle_{L}}{\partial x_{i}}\right) \mathrm{d} t \\
& -\frac{2}{3} \frac{1}{\langle\rho\rangle} \frac{\partial}{\partial x_{i}}\left(\mu \frac{\partial\left\langle u_{j}\right\rangle_{L}}{\partial x_{j}}\right) \mathrm{d} t \\
& +G_{i j}\left(u_{j}^{+}-\left\langle u_{j}\right\rangle_{L}\right) \mathrm{d} t \\
& +\sqrt{C_{0} k \omega} \mathrm{d} W_{i}^{\prime} \\
& +\sqrt{\frac{2 \mu}{\langle\rho\rangle}} \frac{\partial\left\langle u_{i}\right\rangle_{L}}{\partial x_{j}} \mathrm{~d} W_{j}, \\
\mathrm{~d} \phi_{\alpha}^{+} & =-C_{\phi} \omega\left(\phi_{\alpha}^{+}-\left\langle\phi_{\alpha}\right\rangle_{L}\right) \mathrm{d} t \\
& +S_{\alpha}\left(\phi^{+}\right) \mathrm{d} t,
\end{aligned}
$$

where $x_{i}^{+}, u_{i}^{+}, \psi_{\alpha}^{+}$are probabilistic representations of position, velocity vector and scalar variables, respectively. $\mu$ denotes the fluid dynamic viscosity. The $W$ terms denote the Wiener-Lévy processes in the physical (without the prime) and the velocity (with prime) spaces Gardiner (1990). The terms $G_{i j}$ and $k=T_{i i} / 2\langle\rho\rangle$ denote the kernel of GLM and the SGS kinetic energy, respectively. The mixing frequency $\omega$ is defined as: $\omega=C_{f} k^{1 / 2} / \Delta_{\mathcal{H}}$. The constants: $C_{0}, C_{\phi}$ and $C_{f}$ are model parameters and are chosen as suggested in the literature Pope (1994a). The corresponding Fokker-Planck equation, or effectively the modeled VS-FMDF transport equation becomes:

$$
\begin{aligned}
\frac{\partial \mathcal{F}}{\partial t}+\frac{\partial v_{i} \mathcal{F}}{\partial x_{i}} & =\frac{1}{\langle\rho\rangle} \frac{\partial\langle p\rangle}{\partial x_{i}} \frac{\partial \mathcal{F}}{\partial v_{i}} \\
& -\frac{2}{\langle\rho\rangle} \frac{\partial}{\partial x_{j}}\left(\mu \frac{\partial\left\langle u_{i}\right\rangle_{L}}{\partial x_{j}}\right) \frac{\partial \mathcal{F}}{\partial v_{i}} \\
& -\frac{1}{\langle\rho\rangle} \frac{\partial}{\partial x_{j}}\left(\mu \frac{\partial\left\langle u_{j}\right\rangle_{L}}{\partial x_{i}}\right) \frac{\partial \mathcal{F}}{\partial v_{i}} \\
& +\frac{2}{3} \frac{1}{\langle\rho\rangle} \frac{\partial}{\partial x_{i}}\left(\mu \frac{\partial\left\langle u_{j}\right\rangle_{L}}{\partial x_{j}}\right) \frac{\partial \mathcal{F}}{\partial v_{i}} \\
& -G_{i j} \frac{\partial\left[\left(v_{j}-\left\langle u_{j}\right\rangle_{L}\right) \mathcal{F}\right]}{\partial v_{i}} \\
& +\frac{\partial}{\partial x_{j}}\left(\mu \frac{\partial(\mathcal{F} /\langle\rho\rangle)}{\partial x_{j}}\right) \\
& +\frac{\partial}{\partial x_{j}}\left(\frac{2 \mu}{\langle\rho\rangle} \frac{\partial\left\langle u_{i}\right\rangle_{L}}{\partial x_{j}} \frac{\partial \mathcal{F}}{\partial v_{i}}\right) \\
& +\frac{\mu}{\langle\rho\rangle} \frac{\partial\left\langle u_{i}\right\rangle_{L}}{\partial x_{k}} \frac{\left.\partial u_{j}\right\rangle_{L}}{\partial x_{k}} \frac{\partial^{2} \mathcal{F}}{\partial v_{i} \partial v_{j}} \\
& +\frac{1}{2} C_{0} k \omega \frac{\partial^{2} \mathcal{F}}{\partial v_{i} \partial v_{i}} \\
& +C_{\phi} \omega \frac{\partial\left[\left(\psi_{\alpha}-\left\langle\phi_{\alpha}\right\rangle_{L}\right) \mathcal{F}\right]}{\partial \psi_{\alpha}}
\end{aligned}
$$

$$
-\frac{\partial\left[S_{\alpha}(\psi) \mathcal{F}\right]}{\partial \psi_{\alpha}}
$$

\section{LAgRANGiAn FDF SimUlator}

For the FDF to be practical, it must be implemented in a computationally efficient manner, especially if it is to be employed for the prediction of complex flows. For example, some of the current LES methods require up to several months of computing time for a moderately sized domain! In many cases, this is simply due to inefficient usage of a computer's resources. With petascale computing becoming a reality, one must take advantage of the enormous opportunities it provides for FDF simulations.

An effective way to solve the FDF numerically is via Lagrangian Monte Carlo (MC) methods (Grigoriu 1995; Kloeden et al. 1997). These methods have been the primary means of solving the PDF in RANS and, thus far, the most effective choice for solving the FDF in LES (including all of the previous works cited in $\S 1$ ). There have been some efforts to resurrect Eulerian PDF solvers (Chen 2007; Sabel'nikov and Soulard 2005), but they have not shown any particular advantages over Lagrangian methods. See Madnia et al. (2006) for a complete discussion. In the Lagrangian setting, the physical domain is discretized in standard formats (via finite difference, finite volume, spectral, or other methods), and the FDF is represented by an ensemble of particles. Each of these particles carry information pertaining to the physical field (i.e. each of the EPFVS variables) and also the position vector. This is shown in Fig. 1 in which the MC particles are overlaid on grid points, and the transport properties are identified by the colors of the particles. These properties are updated via temporal integration of the modeled SDEs.

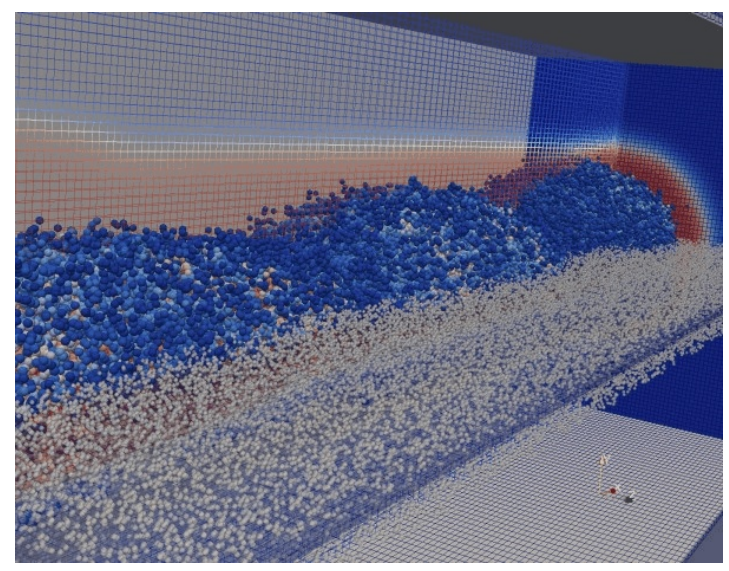

Fig. 1. Lagrangian Monte Carlo particles over structured Eulerian grids. The colors of the particles denote their transport properties. Displayed are particles on the center plane, and within a mixture fraction isosurface.

MC simulations typically require on the order of millions to billions of particles. The computational requirements can become "monstrous" for LES of practical flows, especially those involving complex kinetics. Therefore, scalable parallelization at the MC particle level is required. The major challenge in scalability is the extreme load imbalance associated with stiff chemistry. At any time during the simulation, different regions of the flow 

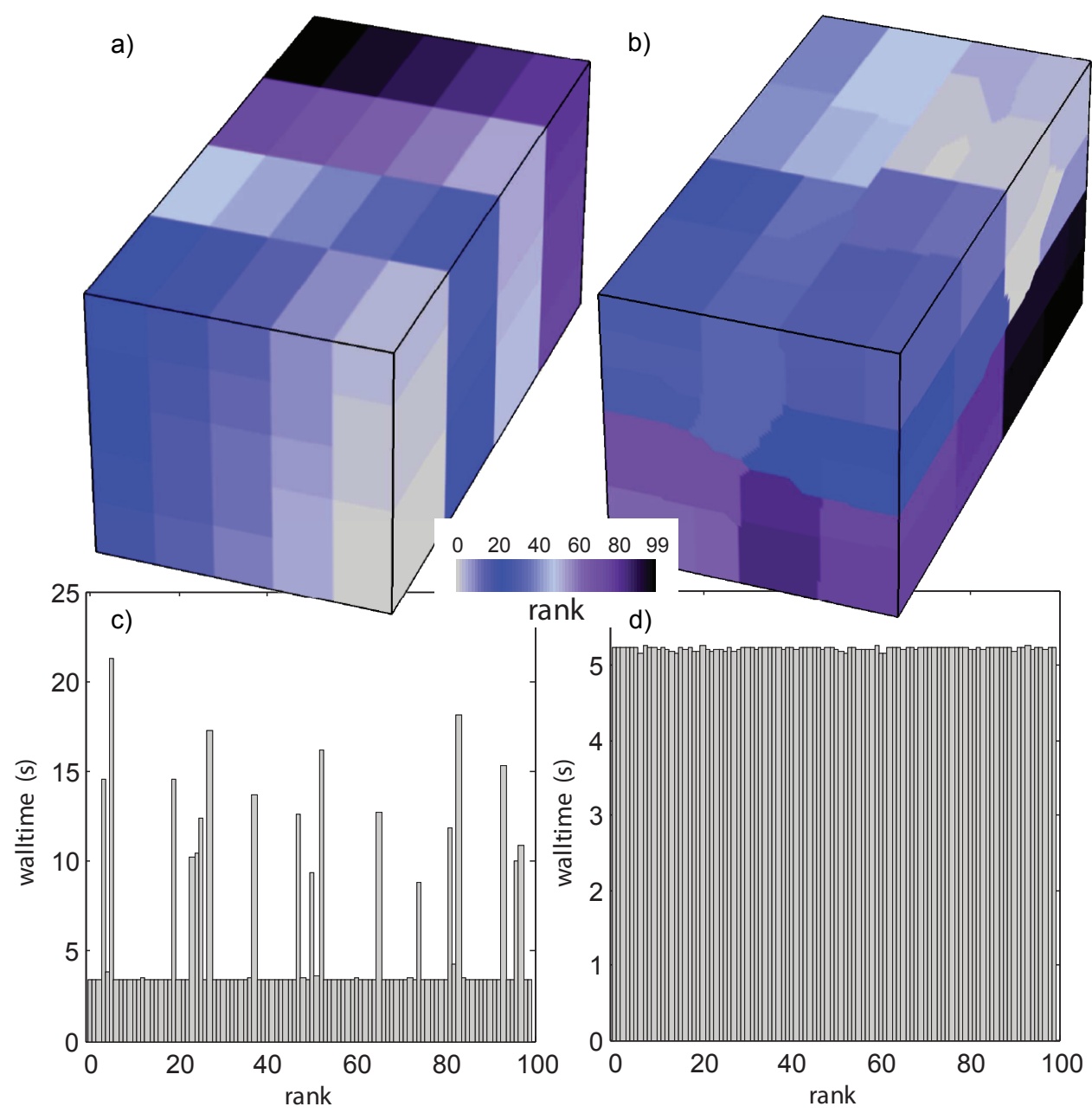

Fig. 2. Domain topology with (a) the uniform decomposition, and (b) adaptive irregular decomposition. Non-idle CPU times per time step for each rank for subsequent time steps with (c) uniform decomposition, and (d) adaptive irregular decomposition.

experience different stages of chemical reactions. Even though the particle number density is statistically uniform spatially, the computational load per particle varies significantly.

A popular parallelization strategy in modern CFD is via temporally invariant block decomposition, where the mesh is partitioned into equally sized boxes, and each box is assigned to a processor (Fig. 2a). This uniformity is relatively easy to implement and yields a minimal communication overhead, but for unsteady and inhomogeneous flows, it usually leads to a poor load distribution. Processors with lighter loads must wait (and remain idle) until the synchronization at the end of each time step. The local computation time of each processor for this is shown in Fig. 2(c). In this example, the idle time is about half of the total time! The load imbalance problem is resolved by development of an irregularly portioned Lagrangian Monte Carlo solver (IPLMC) Yilmaz et al. (2011). In this method, the Eulerian mesh is represented as an undirected graph where particle cells are the vertices of the graph and are weighted by the computational load. Each vertex is assigned a computational weight, i.e. a computation-load metric, which is a function of heterogeneous and homogeneous computational loads. This weighted graph is then fed into a graph partitioning algorithm (Karypis and Kumar 1999) which subdivides the domain into clusters of particle cells on which the computational load is evenly distributed. Figure 2(d) shows the load distribution corresponding to the balanced irregular decomposition. Here, the idle time is insignificant. The transient load redistribution problem is resolved by recomputing the load metric, repartitioning the domain, and intercommunicating the local data of the partitions as the simulation proceeds. The frequency of redistribution is adjusted based on the communication cost and the extent of load imbalance.

In its current implementation, LES/FDF employs MPI for inter-domain communication, and can scale up to $1000 \mathrm{~s}$ of segments via the adaptive partitioning as described. Further local refinement can be made at each individual domain, where ensembles of MC particles can be assigned to accelerator units for simultaneous computations. Sample results to demonstrate scalability are presented in Fig. 3. Shown on the left is the ratio of sequential wall time $\left(t_{1}\right)$ to wall-time with $p$ CPU cores $\left(t_{p}\right)$, i.e. the speed-up (or strong scaling). On the right is the scalability (or weak scaling); that is the ratio of total computational work per time for $p$ CPU cores $\left(N_{p} / t_{p}\right)$ to that with 


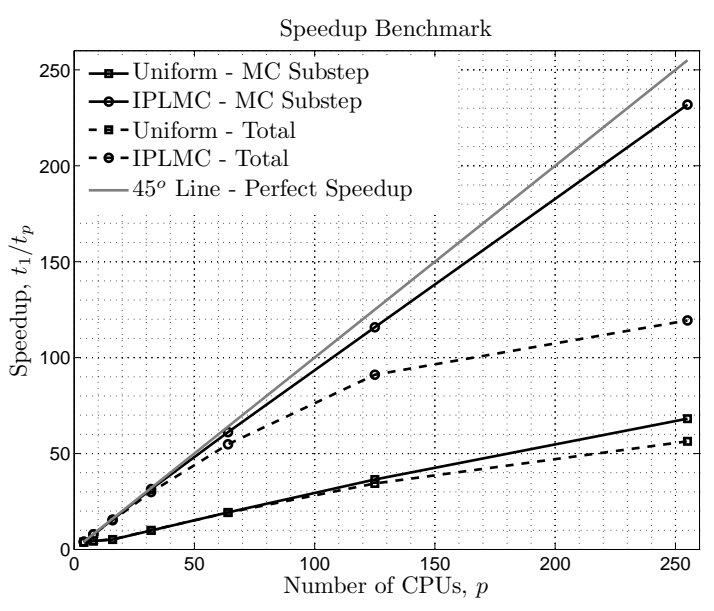

(a)

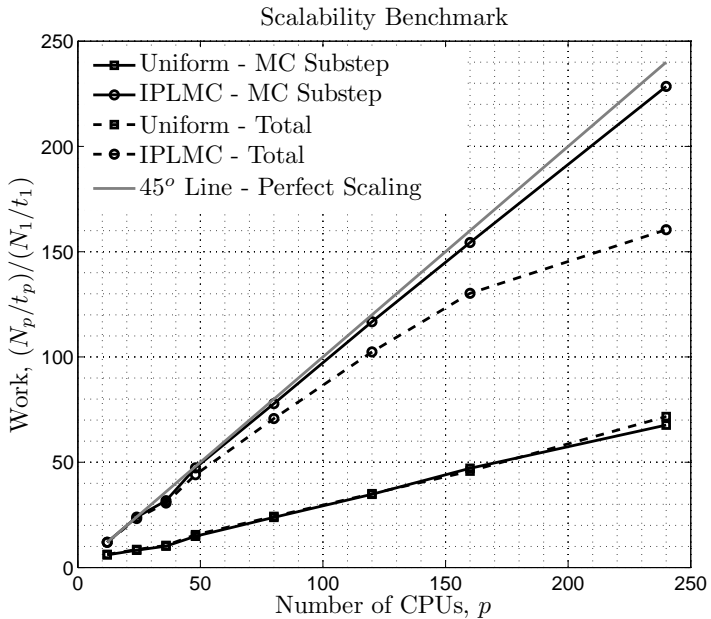

(b)

Fig. 3. Speed-up (strong scaling) and scalability (weak scaling) comparisons of the IPLMC versus uniform decomposition, based on wall-clock timings for chemistry substep and total iteration step. $N_{p}$ denotes the total number of MC particles, and is increased linearly with number of CPUs $(p)$ for the scalability benchmark.

a single core $\left(N_{1} / t_{1}\right)$. In this analysis, the Eulerian mesh is kept fixed, but the number of MC particles per mesh point is increased in direct proportion to $p$. In the speedup analysis, only $p$ is varied for the same mesh with a fixed number of MC particles. In Fig. 3, the MC substep curves (solid) are for that subset of the computation, and message passing is done by the particle solver (including particle migration). The label 'total' (dashed) refers to these and everything else within the whole time step. As seen in both the speed-up and scalability curves, the IPLMC performs better, and is nearly perfect in scaling up-to $250 \mathrm{CPU}$ cores for the MC solver. However, scaling to much larger number of CPUs is less impressive. This is not surprising, because with more CPUs no matter how well the load is balanced at the particle level, overall parallel performance is constrained by the sequential flow solver limiting the scalability as governed by Amdahl's law Amdahl (1967). The time spent on the parallel (MC) and sequential parts of the simulation are shown in Fig. 4. The MC substep time is sharply reduced in IPLMC; and after 150 or so CPU cores, the sequential parts start dominating the computation. With uniform decomposition, this ratio does not decrease nearly as much, agreeing with the observation made in Fig. 3 where the scalability characteristic of the total simulation is not very different than that in the MC substep. Future work includes the full parallelization by a similar strategy for the Eulerian solver, and aggressively reducing the sequential parts of the implementation. As demonstrated for the perfect scaling in the MC substep, this may facilitate near perfect scaling up to 1000 s of CPU cores.

\section{UnSTRUCTURED FDF SOLVER}

The FDF and its IPLMC (or other MC) simulator(s) must be coupled with a base flow solver which identifies the computational domain. A major challenge in doing so is the discretization of complex geometries. Structured grids, as are typically used, lack the required flexibility and robustness for handling such geometries. Also, the
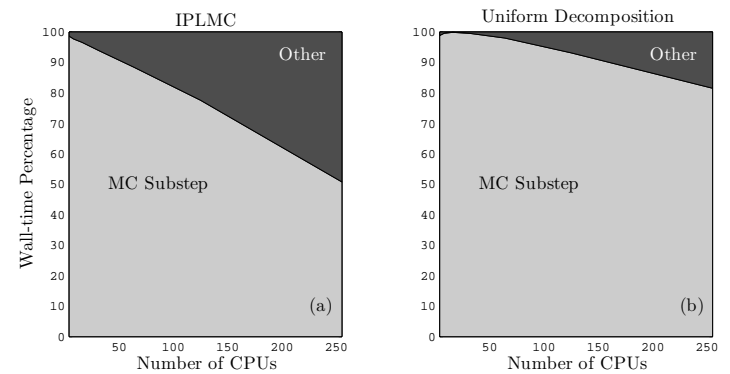

Fig. 4. Percentages of computational work for the Monte Carlo solver and the rest of the simulation for different CPU counts.

grid cells may become too skewed and/or twisted, prohibiting efficient simulations. Unstructured grids provide an excellent remedy for the problem of producing grids for complex geometries. Such grids have irregularly distributed nodes and their cells are not required to be of a specific shape. Furthermore, the connectivity of neighboring cells can vary spatially.

For LES of problems of practical interest, we have developed an unstructured grid system on which the MC simulator can be constructed Ansari et al. (2011). The Eulerian flow solver is based on a second order finite-volume (FV) method. The sacrifice for this lower discretization accuracy, as compared to that of high-order finite difference methods, is justified by the versatility provided by the FV method for consideration of complex configurations. The computational domain is discretized via cells of arbitrary shapes. These cells serve two purposes: (1) they identify the regions where the statistical information from the MC are obtained; (2) they are used to couple the $\mathrm{FV}$ and MC solvers for LES. The FV solver is primarily based on a bounded central composite normalized variable diagram (NVD) scheme Darwish (1993), and consists of a blended scheme of central differencing and a second order upwind scheme. The statistics are gener- 
ated by consideration of the MC particles within a volume centered at the point of interest. Effectively, this volume constitutes an "ensemble domain" characterized by the length scale $\Delta_{E}$ in which the FDF is discretely represented. For reliable statistics with minimal dispersion, it is required to maximize the number of particles and to minimize the size of the ensemble domain. In most of our work, the tetrahedral cell is taken as the ensemble domain. To maximize accuracy with a finite number of particles, a variant of the "basis function method" (Subramaniam and Haworth 2000) is considered. Solution of the modeled stochastic differential equation (7) requires the input of the filtered velocity, the diffusion coefficient and gradients of the scalars field. These are provided by the FV solution and are subsequently interpolated to the particles' locations via a piecewise linear reconstruction method (Barth and Jespersen 1989). Within the cell, the filtered values are expressed in terms of the vertex mean values. In doing so, an efficient particle tracking procedure is constructed Ansari et al. (2011).

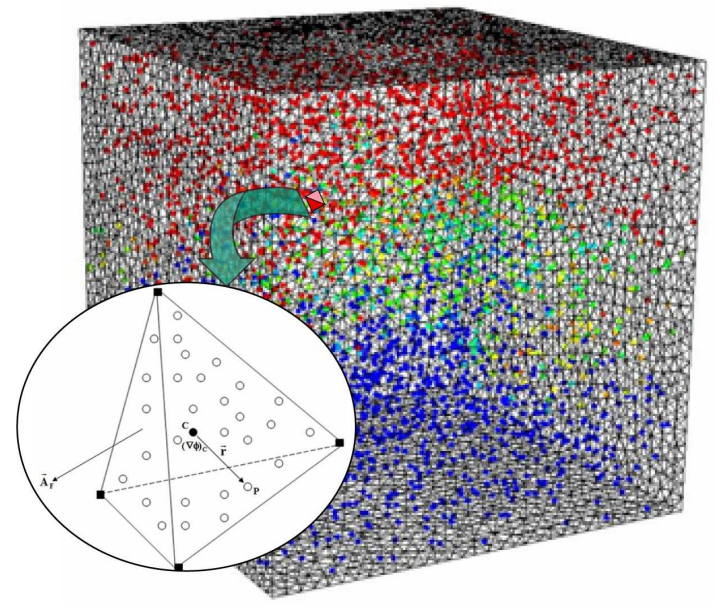

Fig. 5. Lagrangian Monte Carlo particles over unstructured Eulerian tetrahedrons for LES of a homogeneous turbulent flow Sheikhi et al. (2009). The colors of the particles denote their transport properties.

We demonstrate the feasibility of the method by applying our S-FMDF for LES of a homogeneous turbulent flow. Figure 5 shows an instantaneous ensemble of MC particles overlaid on an unstructured grid. The inset shows the schematics of a tetrahedral cell, and the MC particles it contains. The success of this new solver is assessed by duplicating our previous results via a high-order finite difference algorithm Sheikhi et al. (2009). We have implemented the solver for LES of a variety of turbulent flames. The most complex case considered thus far is the PRECCINSTA experimental burner from the German Aerospace Center (DLR) Meier et al. (2007). This is an excellent representation of an industrial gas turbine burner. The mesh for this burner is shown in Fig. 6. It consists of 2.2 million tetrahedral unstructured mesh elements. Figure 7 shows the volume rendered, instantaneous $\mathrm{CO}$ mass fraction. The flame with a $\mathrm{V}$-shape is located near the burner inlet and shows wrinkling in its structure. The structure of the turbulent flow field is ev- ident in the isosurface of the second invariant of the velocity deformation tensor colored by velocity as shown in Fig. 8 .

\section{Concluding Remarks}

The filtered density function (FDF) has proven very effective for reliable and affordable prediction of turbulent reactive flows. The primary advantage of the FDF is that it accounts for the effects of subgrid-scale (SGS) chemical reactions in an exact manner regardless of the speed of reaction. Also, it provides all the higher SGS moments of the flow; whereas most other LES strategies predict only the mean values, at best. Since its original development, the FDF has experienced widespread applications for LES of a variety of reacting flows, as reviewed in this article. The methodology has been the subject of broad coverage in modern text- and hand-books (Bilger 2000; Fox 2003; Heinz 2003a; Kuo and Acharya 2012; Minkowycz et al. 2006; Peters 2000; Pope 2000). It has been implemented in commercial and government combustion codes such as FLUENT/ANSYS Ansari et al. (2011), VULCAN Drozda et al. (2012), and US3D Otis et al. (2012), among others.

Recent developments in FDF simulation consist of (1) construction of an irregularly portioned Lagrangian Monte Carlo FDF solver and (2) implementation of (any) Monte Carlo simulator on Eulerian domains portrayed by unstructured grids. The former allows efficient FDF simulations on massively parallel platforms, and the latter facilitates discretization of flows in complex geometrical configurations. With these developments, it is now possible to apply FDF for LES of practical problems, such as gas-turbine combustors.

\section{ACKNOWLEDGMENTS}

Our current FDF related research is sponsored by the AFOSR \& NASA under Grant FA9550-09-1-0611, by AFOSR under Grant FA9550-12-1-0057, by NSF under Grant CBET-1250171, by DOE under contract RES10000027/96D, by the NSF Extreme Science and Engineering Discovery Environment (XSEDE) under Grants TG-CTS070055N and TG-CTS120015, and by the University of Pittsburgh Center for Simulation and Modeling.

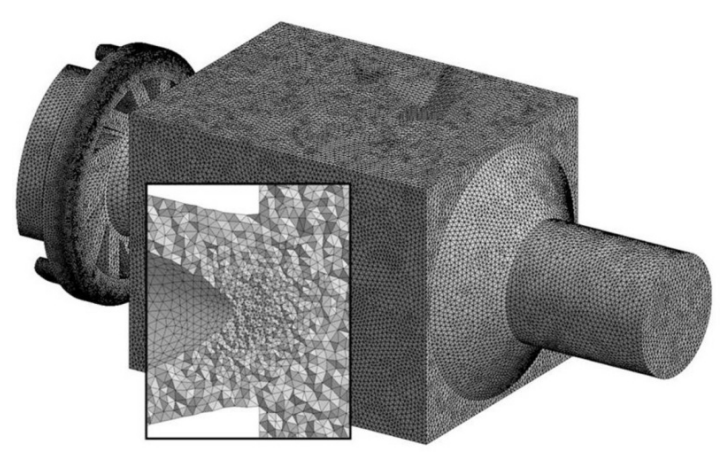

Fig. 6. Tetrahedral mesh on the PRECCINSTA burner Meier et al. (2007) and combustion chamber. 


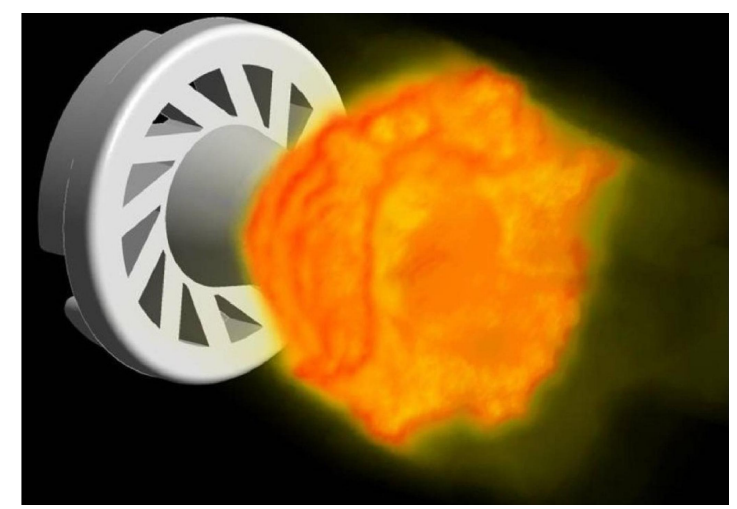

Fig. 7. Instantaneous volume rendered plot of $\mathrm{CO}$ mass fraction.

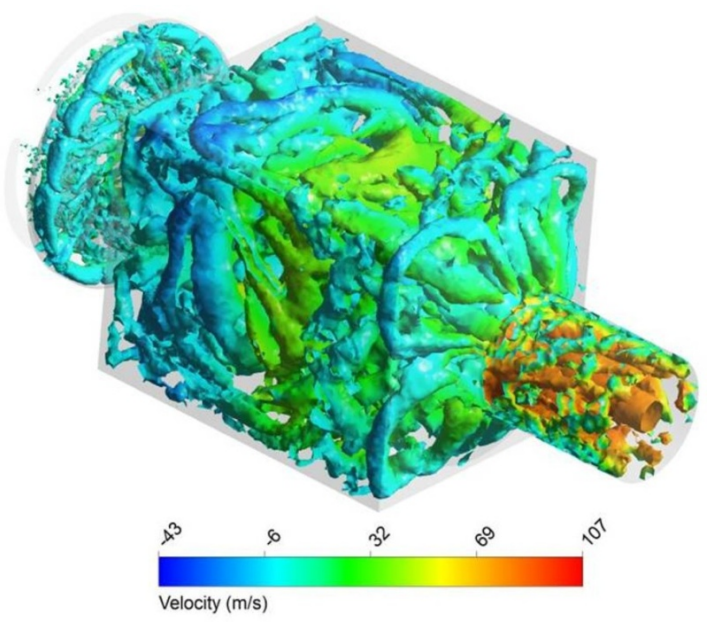

Fig. 8. Flow structure for PRECCINSTA burner (Meier et al. 2007) visualized by the isosurface of the second invariant of the velocity deformation tensor colored by velocity.

\section{REFERENCES}

Afshari, A., F. Jaberi, and T. Shih (2008). Large-Eddy Simulations of Turbulent Flows in an Axisymmetric Dump Combustor. AIAA J. 46(7), 1576-1592.

Amdahl, G. M. (1967). Validity of the Single Processor Approach to Achieving Large Scale Computing Capabilities. In Proceedings of the April 18-20, 1967, Spring Joint Computer Conference, AFIPS '67 (Spring), New York, NY, pp. 483-485.

Ansari, N. (2012). Filtered Density Function for Large Eddy Simulation of Turbulent Reacting Flows on Unstructured Meshes. Ph.D. Thesis, Department of Mechanical Engineering and Materials Science, University of Pittsburgh, Pittsburgh, PA.

Ansari, N., G. M. Goldin, P. H. Pisciuneri, M. B. Nik, P. A. Strakey, and P. Givi (2011). FDF Simulation of Swirling Reacting Flows on Unstructured Meshes. In 49th AIAA Aerospace Sciences Meeting including the New Horizons Forum and Aerospace Exposition, Orlando, FL, pp. 1-17. AIAA-2011-109.
Ansari, N., G. M. Goldin, M. R. H. Sheikhi, and P. Givi (2011). Filtered Density Function Simulator on Unstructured Meshes. J. Comput. Phys. 230(19), 71327150 .

Ansari, N., F. A. Jaberi, M. R. H. Sheikhi, and P. Givi (2011). Filtered Density Function as a Modern CFD Tool. In A. R. S. Maher (Ed.), Engineering Applications of Computational Fluid Dynamics: Volume 1, Chapter 1, pp. 1-22. International Energy and Environment Foundation.

Barlow, R. S. and J. H. Frank (1998). Effects of Turbulence on Species Mass Fractions in Methane/Air Jet Flames. Proc. Combust. Inst. 27(1), 1087-1095.

Barth, T. J. and D. C. Jespersen (1989). The Design and Application of Upwind Schemes on Unstructured Meshes. In 27th Aerospace Sciences Meeting, Reno, NV, pp. 1-12. AIAA-1989-0366.

Bilger, R. W. (2000). Future Progress in Turbulent Combustion Research. Prog. Energ. Combust. 26(4-6), 367-380.

Borghi, R. (1988). Turbulent Combustion Modelling. Prog. Energ. Combust. 14(4), 245-292.

Carrara, M. D. and P. E. DesJardin (2006). A Filtered Mass Density Function Approach for Modeling Separated Two-Phase Flows for LES I: Mathematical Formulation. Int. J. Multiphas. Flow 32(3), 365-384.

Cha, C. M. and P. Trouillet (2003). A Subgrid-Scale Mixing Model for Large-Eddy Simulation of Turbulent Reacting Flows using the Filtered Density Function. Phys. Fluids 15(6), 1496-1504.

Chen, J.-Y. (2007). A Eulerian PDF Scheme for LES of Nonpremixed Turbulent Combustion with SecondOrder Accurate Mixture Fraction. Combust. Theor. Model. 11(5), 675-695.

Chen, Y.-C., N. Peters, G. A. Schneemann, N. Wruck, U. Renz, and M. S. Mansour (1996). The Detailed Flame Structure of Highly Stretched Turbulent Premixed Methane-Air Flames. Combust. Flame 107(3), 223-226.

Colucci, P. J. (1998). Large Eddy Simulation of Turbulent Reactive Flows: Stochastic Representation of the Subgrid Scale Scalar Fluctutations. Ph.D. Thesis, State University of New York at Buffalo, Buffalo, NY.

Colucci, P. J., F. A. Jaberi, P. Givi, and S. B. Pope (1998). Filtered Density Function for Large Eddy Simulation of Turbulent Reacting Flows. Phys. Fluids 10(2), 499515.

Dally, B. B., A. R. Masri, R. S. Barlow, and G. J. Fiechtner (1998). Instantaneous and Mean Compositional Structure of Bluff-Body Stabilized Nonpremixed Flames. Combust. Flame 114(1-2), 119148.

Dally, B. B., A. R. Masri, R. S. Barlow, and G. J. Fiechtner (2003). Two-Photon Laser-Induced Fluorescence Measurement of CO in Turbulent Non-Premixed Bluff Body Flames. Combust. Flame 132(1-2), 272-274. 
Darwish, M. S. (1993). A New High Resolution Scheme Based on the Normalized Variable Formulation. $\mathrm{Nu}$ mer. Heat Tr. B-Fund. 24(3), 353-371.

Dopazo, C. (1994). Recent Developments in PDF Methods. In P. A. Libby and F. A. Williams (Eds.), Turbulent Reacting Flows, Chapter 7, pp. 375-474. London, UK: Academic Press.

Dreeben, T. D. and S. B. Pope (1997). Probability Density Function and Reynolds-Stress Modeling of NearWall Turbulent Flows. Phys. Fluids 9(1), 154-163.

Drozda, T. G. (2005). Implementation of LES/SFMDF for Prediction of Non-Premixed Turbulent Flames. Ph.D. Thesis, Department of Mechanical Engineering, University of Pittsburgh, Pittsburgh, PA.

Drozda, T. G., J. R. Quinlan, P. H. Pisciuneri, and S. L. Yilmaz (2012). Progress Toward Affordable High Fidelity Combustion Simulations for HighSpeed Flows in Complex Geometries. In 48th AIAA/ASME/SAE/ASEE Joint Propulsion Conference \& Exhibit, Atlanta, GA, pp. 1-16. AIAA-2012-4264.

Drozda, T. G., M. R. H. Sheikhi, C. K. Madnia, and P. Givi (2007). Developments in Formulation and Application of the Filtered Density Function. Flow Turbul. Combust. 78(1), 35-67.

Drozda, T. G., G. Wang, V. Sankaran, J. R. Mayo, J. C. Oefelein, and R. S. Barlow (2008). Scalar Filtered Mass Density Functions in Nonpremixed Turbulent Jet Flames. Combust. Flame 155(1-2), 54-69.

Fox, R. O. (2003). Computational Models for Turbulent Reacting Flows. Cambridge, UK: Cambridge University Press.

Gardiner, C. W. (1990). Handbook of Stochastic Methods for Physics, Chemistry and the Natural Sciences (Second ed.). New York, NY: Springer-Verlag.

Garrick, S. C., F. A. Jaberi, and P. Givi (1999). Large Eddy Simulation of Scalar Transport in a Turbulent Jet Flow. In D. Knight and L. Sakell (Eds.), Recent Advances in DNS and LES, Volume 54 of Fluid Mechanics and its Applications, pp. 155-166. Springer Netherlands.

Geurts, B. J. (2004). Elements of Direct and Large-Eddy Simulation. Philadelphia, PA: R. T. Edwards, Inc.

Ghosal, S. and P. Moin (1995). The Basic Equations for the Large Eddy Simulation of Turbulent Flows in Complex Geometry. J. Comput. Phys. 118(1), 24-37.

Gicquel, L. Y. M. (2001). Velocity Filtered Density Function for Large Eddy Simulation of Turbulent Flows. Ph.D. Thesis, State University of New York at Buffalo, Buffalo, NY.

Gicquel, L. Y. M., P. Givi, F. A. Jaberi, and S. B. Pope (2002). Velocity Filtered Density Function for Large Eddy Simulation of Turbulent Flows. Phys. Fluids 14(3), 1196-1213.
Gikhman, I. I. and A. V. Skorokhod (1972). Stochastic Differential Equations. New York, NY: SpringerVerlag.

Givi, P. (2006). Filtered Density Function for Subgrid Scale Modeling of Turbulent Combustion. AIAA J. 44(1), 16-23.

Grigoriu, M. (1995). Applied Non-Gaussian Processes. Englewood Cliffs, NJ: Prentice-Hall.

Haworth, D. C. and S. B. Pope (1986). A Generalized Langevin Model for Turbulent Flows. Phys. Fluids 29(2), 387-405.

Heinz, S. (2003a). On Fokker-Planck Equations for Turbulent Reacting Flows. Part 1. Probability Density Function for Reynolds-Averaged Navier-Stokes Equations. Flow Turbul. Combust. 70(1-4), 115-152.

Heinz, S. (2003b). On Fokker-Planck Equations for Turbulent Reacting Flows. Part 2. Filter Density Function for Large Eddy Simulation. Flow Turbul. Combust. 70(1-4), 153-181.

Heinz, S. (2007). Unified Turbulence Models for LES and RANS, FDF and PDF Simulations. Theor. Comp. Fluid Dyn. 21(2), 99-118.

Jaberi, F. A., P. J. Colucci, S. James, P. Givi, and S. B. Pope (1999). Filtered Mass Density Function for Large-Eddy Simulation of Turbulent Reacting Flows. J. Fluid Mech. 401, 85-121.

James, S., J. Zhu, and M. S. Anand (2007). Large Eddy Simulation of Turbulent Flames Using the Filtered Density Function Model. Proc. Combust. Inst. 31(2), 1737-1745.

Jones, W. P. and S. Navarro-Martinez (2007). Large Eddy Simulation of Autoignition with a Subgrid Probability Density Function Method. Combust. Flame 150(3), 170-187.

Jones, W. P., S. Navarro-Martinez, and O. Rohl (2007). Large Eddy Simulation of Hydrogen Auto-Ignition with a Probability Density Function Method. Proc. Combust. Inst. 31(2), 1765-1771.

Karlin, S. and H. M. Taylor (1981). A Second Course in Stochastic Processes. New York, NY: Academic Press.

Karypis, G. and V. Kumar (1999). A Fast and High Quality Multilevel Scheme for Partitioning Irregular Graphs. SIAM J. Sci. Comput. 20(1), 359-392.

Kloeden, P. E., E. Platen, and H. Schurz (1997). Numerical Solution of Stochastic Differential Equations through Computer Experiments (Corr. 2nd print ed.). Berlin; New York: Springer.

Kuo, K. K. and R. Acharya (2012). Fundamentals of Turbulent and Multiphase Combustion. Hoboken, NJ: John Wiley and Sons Inc.

Madnia, C. K., F. A. Jaberi, and P. Givi (2006). Large Eddy Simulation of Heat and Mass Transport in Turbulent Flows. See Minkowycz et al. (2006), Chapter 5, pp. 167-190. 
McDermott, R. and S. B. Pope (2007). A Particle Formulation for Treating Differential Diffusion in Filtered Density Function Methods. J. Comput. Phys. 226(1), 947-993.

Meier, W., P. Weigand, X. R. Duan, and R. GiezendannerThoben (2007). Detailed Characterization of the Dynamics of Thermoacoustic Pulsations in a Lean Premixed Swirl Flame. Combust. Flame 150(1-2), 2-26.

Minkowycz, W. J., E. M. Sparrow, and J. Y. Murthy (Eds.) (2006). Handbook of Numerical Heat Transfer (Second ed.). New York, NY: Wiley.

Mustata, R., L. Valiño, C. Jiménez, W. Jones, and S. Bondi (2006). A Probability Density Function Eulerian Monte Carlo Field Method for Large Eddy Simulations: Application to a Turbulent Piloted Methane/Air Diffusion Flame (Sandia D). Combust. Flame 145(1-2), 88-104.

Nik, M. B. (2012). VS-FMDF and EPVS-FMDF for Large Eddy Simulation of Turbulent Flows. Ph.D. Thesis, Department of Mechanical Engineering and Materials Science, University of Pittsburgh, Pittsburgh, PA.

Nik, M. B., S. L. Yilmaz, P. Givi, M. R. H. Sheikhi, and S. B. Pope (2010). Simulation of Sandia Flame D Using Velocity-Scalar Filtered Density Function. AIAA J. 48(7), 1513-1522.

Nik, M. B., S. L. Yilmaz, M. R. Sheikhi, and P. Givi (2010). Grid Resolution Effects on VSFMDF/LES. Flow Turbul. Combust. 85(3-4), 677-688.

Otis, C. C., P. Ferrero, S. L. Yilmaz, G. V. Candler, and P. Givi (2012). SFMDF in US3D for LES of Compressible Flows on Unstructured Meshes. In 48th AIAA/ASME/SAE/ASEE Joint Propulsion Conference \& Exhibit, Atlanta, GA, pp. 1-11. AIAA-2012-4260.

Peters, N. (2000). Turbulent Combustion. Cambridge, UK: Cambridge University Press.

Pisciuneri, P. H. (2008). Large Eddy Simulation of a Turbulent Nonpremixed Jet Flame Using a Finite-Rate Chemistry Model. M.S. Thesis, Department of Mechanical Engineering and Materials Science, University of Pittsburgh, Pittsburgh, PA.

Pope, S. B. (1994a). Lagrangian PDF Methods for Turbulent Flows. Annu. Rev. Fluid Mech. 26, 23-63.

Pope, S. B. (1994b). On the Relation Between Stochastic Lagrangian Models of Turbulence and SecondMoment Closures. Phys. Fluids 6(2), 973-985.

Pope, S. B. (2000). Turbulent Flows. Cambridge, U.K.: Cambridge University Press.

Pope, S. B. (2013). Small Scales, Many Species and the Manifold Challenges of Turbulent Combustion. Proc. Combust. Inst. 34(1), $1-31$.

Rajagopalan, A. G. and C. Tong (2003). Experimental Investigation of Scalar-Scalar-Dissipation Filtered Joint Density Function and its Transport Equation. Phys. Fluids 15(1), 227-244.
Raman, V. and H. Pitsch (2005). Large-Eddy Simulation of a Bluff-Body-Stabilized Non-Premixed Flame using a Recursive Filter-Refinement Procedure. Combust. Flame 142(4), 329-347.

Raman, V. and H. Pitsch (2007). A Consistent LES/Filtered-Density Function Formulation for the Simulation of Turbulent Flames with Detailed Chemistry. Proc. Combust. Inst. 31(2), 1711-1719.

Raman, V., H. Pitsch, and R. O. Fox (2005). Hybrid Large-Eddy Simulation/Lagrangian Filtered-DensityFunction Approach for Simulating Turbulent Combustion. Combust. Flame 143(1-2), 56-78.

Réveillon, J. and L. Vervisch (1998). Subgrid-Scale Turbulent Micromixing: Dynamic Approach. AIAA J. 36(3), 336-341.

Risken, H. (1989). The Fokker-Planck Equation, Methods of Solution and Applications. New York, NY: Springer-Verlag.

Sabel'nikov, V. and O. Soulard (2005). Rapidly Decorrelating Velocity-Field Model as a Tool for Solving One-Point Fokker-Planck Equations for Probability Density Functions of Turbulent Reactive Scalars. Phys. Rev. E 72(1), 016301.

Sagaut, P. (2010). Large Eddy Simulation for Incompressible Flows (Third ed.). New York, NY: Springer.

Sandia National Laboratories (2013). TNF Workshop Abstract. http://www. sandia.gov/TNF/.

Sheikhi, M. R. H. (2005). Joint Velocity Scalar Filtered Density Function for Large Eddy Simulation of Turbulent Reacting Flows. Ph.D. Thesis, Department of Mechanical Engineering, University of Pittsburgh, Pittsburgh, PA.

Sheikhi, M. R. H., T. G. Drozda, P. Givi, F. A. Jaberi, and S. B. Pope (2005). Large Eddy Simulation of a Turbulent Nonpremixed Piloted Methane Jet Flame (Sandia Flame D). Proc. Combust. Inst. 30(1), 549-556.

Sheikhi, M. R. H., T. G. Drozda, P. Givi, and S. B. Pope (2003). Velocity-Scalar Filtered Density Function for Large Eddy Simulation of Turbulent Flows. Phys. Fluids 15(8), 2321-2337.

Sheikhi, M. R. H., P. Givi, and S. B. Pope (2007). Velocity-Scalar Filtered Mass Density Function for Large Eddy Simulation of Turbulent Reacting Flows. Phys. Fluids 19(9), 095106.

Sheikhi, M. R. H., P. Givi, and S. B. Pope (2009). Frequency-Velocity-Scalar Filtered Mass Density Function for Large Eddy Simulation of Turbulent Flows. Phys. Fluids 21(7), 075102.

Stratonovich, R. L. (1963). Introduction to the Theory of Random Noise. New York, NY: Gordon and Breach.

Subramaniam, S. and D. C. Haworth (2000). A Probability Density Function Method for Turbulent Mixing and Combustion on Three-Dimensional Unstructured Deforming Meshes. Int. J. Engine Res. 1(2), 171-190. 
Tong, C. (2001). Measurements of Conserved Scalar Filtered Density Function in a Turbulent Jet. Phys. Fluids 13(10), 2923-2937.

van Vliet, E., J. J. Derksen, and H. E. A. van den Akker (2005). Turbulent Mixing in a Tubular Reactor: Assessment of an FDF/LES Approach. AIChE J. 51(3), 725-739.

Vreman, B., B. Geurts, and H. Kuerten (1994). Realizability Conditions for the Turbulent Stress Tensor in Large-Eddy Simulation. J. Fluid Mech. 278, 351-362.

Wang, D. and C. Tong (2002). Conditionally Filtered Scalar Dissipation, Scalar Diffusion, and Velocity in a Turbulent Jet. Phys. Fluids 14(7), 2170-2185.

Wang, D. and C. Tong (2005). Experimental Study of Velocity-Scalar Filtered Joint Density Function for LES of Turbulent Combustion. Proc. Combust. Inst. 30(1), 567-574.

Wang, D., C. Tong, and S. B. Pope (2004). Experimental Study of Velocity Filtered Joint Density Function for Large Eddy Simulation. Phys. Fluids 16(10), 35993613.
Williams, F. A. (1985). Turbulent Combustion. In J. D. Buckmaster (Ed.), The Mathematics of Combustion, Frontiers in Applied Mathematics. Philadelphia, PA: SIAM.

Yilmaz, S. L. (2008). RANS/PDF and LES/FDF for Prediction of Turbulent Premixed Flames. Ph.D. Thesis, Department of Mechanical Engineering and Materials Science, University of Pittsburgh, Pittsburgh, PA.

Yilmaz, S. L., M. B. Nik, P. Givi, and P. A. Strakey (2010). Scalar Filtered Density Function for Large Eddy Simulation of a Bunsen Burner. J. Propul. Power 26(1), 84-93.

Yilmaz, S. L., M. B. Nik, M. R. H. Sheikhi, P. A. Strakey, and P. Givi (2011). An Irregularly Portioned Lagrangian Monte Carlo Method for Turbulent Flow Simulation. J. Sci. Comput. 47(1), 109-125.

Zhou, X. Y. and J. C. F. Pereira (2000). Large Eddy Simulation (2D) of a Reacting Plane Mixing Layer using Filtered Density Function Closure. Flow Turbul. Combust. 64(4), 279-300. 